\title{
Effects of resveratrol on histone expression in human intestinal Caco-2 cells and human breast cancer MCF-7 cells are mediated through the oestrogen receptor and oppose changes observed in ageing mouse gut
}

\author{
F. Alatawi ${ }^{1}$, L. A. Wakeling ${ }^{1}$, J. C. Mathers ${ }^{2}$, J. E. Hesketh ${ }^{1,2}$ and D. Ford ${ }^{1,2}$ \\ ${ }^{1}$ Human Nutrition Research Centre, Institute for Cell and Molecular Biosciences Newcastle University, Newcastle upon \\ Tyne NE2 4HH, UK and ${ }^{2}$ Human Nutrition Research Centre, Institute for Ageing and Health, Newcastle University, \\ Newcastle upon Tyne NE2 4HH, UK
}

Dietary restriction (DR) increases lifespan robustly in diverse species ${ }^{(1)}$. Effects of the dietary polyphenol resveratrol consistent with delayed ageing and/or extension of lifespan have been reported ${ }^{(1)}$. The protein Sirt1 is involved in the longevity response to DR and can be activated by resveratrol. Sirt1 deacetylates a range of cellular substrates that includes histone proteins ${ }^{(1)}$, identifying epigenetic processes as a pathway that may mediate the effects of both DR and dietary resveratrol in delaying ageing and/or extending lifespan.

We investigated the hypothesis that resveratrol brings about histone deacetylation to oppose changes in histone modification that accompany ageing. A secondary hypothesis, based on a degree of structural similarity between resveratrol and $17 \beta$-oestradiol, is that epigenetic effects of resveratrol are mediated through the oestrogen receptor (ER).

Human intestinal Caco-2 cells or human MCF-7 breast cancer cells were treated with resveratrol $(10 \mu \mathrm{M})$ for $72 \mathrm{~h}$ in the presence or absence of the ER antagonist fulvestrant $(0.1 \mu \mathrm{M})$, then semi-purified histone proteins or total cell lysate were analysed by western blotting using an antibody specific for histone 4 (H4) acetylated at Lys16 or using antibodies immunoreactive against $\mathrm{H} 4$ or $\mathrm{H} 3$ irrespective of acetylation status. Where total cell lysate was analysed, blots were probed with an antibody immunoreactive against $\alpha$-tubulin to correct for protein loading/transfer. Where histone proteins were extracted before analysis, equal loading was confirmed by staining a duplicate gel with Coomassie Brilliant Blue. Quantitative data were derived by densitometric quantification of band intensities and are expressed as mean (SEM) normalised to control.

Both anti-H4 antibodies revealed a marked reduction in the $\mathrm{H} 4$ signal after the treatment of Caco-2 cells with resveratrol (0.13 (0.06), $P<0.01, n 3$ for H4-Lys 16, and 0.25 (0.13), $P<0.05, n 3$ for total H4 protein; both based on analysis of total extracted histone protein), indicating that resveratrol treatment reduces $\mathrm{H} 4$ protein expression, rather than affecting acetylation status. Similarly, the intensity of the immunoreactive band detected using the anti-H3 antibody was reduced by treatment of MCF-7 cells with resveratrol $(0.67(0.13), P<0.05$, $n 3$, based on the analysis of total cell protein and corrected based on the signal for $\alpha$-tubulin). This effect of resveratrol was attenuated by the ER antagonist fulvestrant $(1.06(0.03), n$ 3), indicating that resveratrol reduced histone H3 expression via an ER-dependent mechanism. Preliminary data also reveal attenuation by fulvestrant of the reduction in $\mathrm{H} 4$ observed in Caco- 2 cells in response to resveratrol.

In support of our hypothesis that resveratrol affects ageing through reversing ageing-associated changes in histone proteins, higher levels $(P<0.05)$ of $\mathrm{H} 4$ expression were detected by western blotting in the small intestine of old (38 months) C57Bl6 mice (8.01 (2.88), $n$ 3) than in younger (12 months) mice $(1.00(0.62), n 3)$. Similar changes in expression with age were observed for H2A and H2B but H3 levels did not appear to differ between old and young mice.

In summary, our data indicate that resveratrol reduces histone expression in both intestinal and breast cancer cells through an ER-mediated mechanism and that this effect may oppose an accumulation of histone proteins (observed in mouse small intestine) that accompanies ageing. Identification of genes associated with histones that are expressed differentially under conditions of both resveratrol treatment and ageing may reveal novel candidate targets for actions of resveratrol that counteract the effects of ageing and/or increase lifespan.

Funded by BBSRC grant ref. BBE0074571 and support to FA from Tabouk University, Kingdom of Saudi Arabia.

1. Guarente L (2007) Sirtuins in aging and disease. Cold Spring Harb Symp Quant Biol 72, 483-488. 